\title{
Evaluation of ESSENCE in the Cloud Using Meaningful Use Syndromic Surveillance Data
}

\author{
Wayne Loschen*1, William Stephens ${ }^{2}$, Taha Kass-Hout ${ }^{3}$, Miles Stewart ${ }^{1}$, Dave Heinbaugh ${ }^{2}$ \\ and Joseph Lombardo ${ }^{1}$
}

${ }^{1}$ Johns Hopkins University Applied Physics Laboratory, Laurel, MD, USA; ${ }^{2}$ Tarrant County Public Health, Fort Worth, TX, USA; ${ }^{3}$ Public Health Surveillance and Informatics Program Office, Office of Surveillance, Epidemiology, \& Laboratory Services, Centers for Disease Control and Prevention, Atlanta, GA, USA

\section{Objective}

This project represents collaboration among CDC's BioSense Program, Tarrant County Public Health and the ESSENCE Team at the Johns Hopkins University APL. For over six months the Tarrant County Public Health Department has been sending data through the BioSense 2.0 application to a pilot version of ESSENCE on the Amazon GovCloud. This project has demonstrated the ability for local hospitals to send meaningful use syndromic surveillance data to the Internet cloud and provide public health officials tools to analyze the data both using BioSense 2.0 and ESSENCE. The presentation will describe the tools and techniques used to accomplish this, an evaluation of how the system has performed, and lessons learned for future health departments attempting similar projects.

\section{Introduction}

In November of 2011 BioSense 2.0 went live to provide tools for public health departments to process, store, and analyze meaningful use syndromic surveillance data. In February of 2012 ESSENCE was adapted to support meaningful use syndromic surveillance data and was installed on the Amazon GovCloud. Tarrant County Public Health Department agreed to pilot the ESSENCE system and evaluate its performance compared to a local version ESSENCE they currently used. The project determined the technical feasibility of utilizing the Internet cloud to perform detailed public health analysis, necessary changes needed to support meaningful use syndromic surveillance data, and any public health benefits that could be gained from the technology or data.

\section{Methods}

This project investigated database and visualization changes necessary to support meaningful use syndromic surveillance data in ESSENCE. It evaluated the Internet cloud environment and determined the benefits and disadvantages to using this technology as a platform for ESSENCE. This included scalability, performance, and cost analysis of the Internet cloud platform. After using the system for a period of time, the Tarrant County users evaluated the Internet cloud version of the system.

\section{Results}

Many technical adaptations to the ESSENCE system were made to support the new meaningful use syndromic surveillance elements Several optimizations, including a new database schema and cube table structures, were developed to improve performance of ESSENCE in the Internet cloud and incorporating the meaningful use requirements. The Internet cloud platform offered many levels of performance that could alter the ESSENCE user experience. Smaller configurations allowed for 100 concurrent users to experience $16 \mathrm{sec}-$ ond response times, whereas larger configurations supported experiences of 2 second response times.

\section{Conclusions}

Public health departments are dealing with new meaningful use syndromic surveillance data elements and the cost of maintaining local systems. This collaborative team have researched and evaluated tools, technologies, and solutions that can be used throughout the country.

\section{Keywords}

Electronic Medical Records for Public Health; Interoperability; Meaningful Use; Syndromic Surveillance; Internet Cloud

\section{Acknowledgments}

The ESSENCE in the Cloud initiative is supported by the CDC's Division of Notifiable Diseases and Healthcare Information (DNDHI) BioSense Program.

\footnotetext{
*Wayne Loschen

E-mail: wayne.loschen@jhuapl.edu
} 